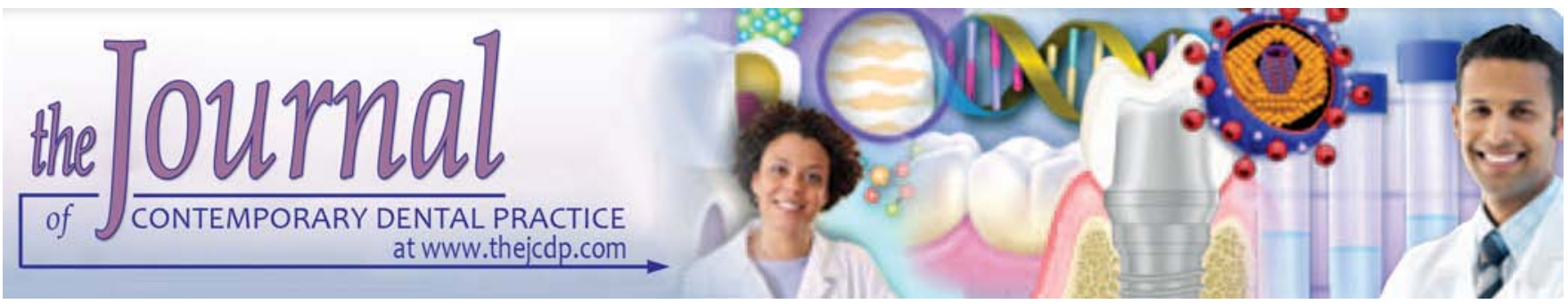

\title{
Management of Impacted Maxillary Central Incisor: Modified Nance Arch Application
}

\author{
${ }^{1}$ Bonolo Mannathoko-Molefhe, ${ }^{2}$ Rongdang Hu
}

\begin{abstract}
This is a case report of a patient who presented to Wenzhou Medical University_Orthodontic Department at 8 years of age with missing right maxillary central incisor. A detailed clinical and radiographic examination was carried out and treatment plan formulated to surgically expose the impacted incisor and use the modified nance arch (MNA) appliance for orthodontic traction of the tooth in line of arch as phase one treatment followed by phase two treatment with fixed appliances.
\end{abstract}

Keywords: Impacted maxillary incisor, Modified nance arch appliance, Orthodontic alignment, Surgical exposure.

How to cite this article: Mannathoko-Molefhe B, Hu R. Management of Impacted Maxillary Central Incisor: Modified Nance Arch Application. J Contemp Dent Pract 2015;16(5): 415-421.

Source of support: This work was supported by grants from the Provincial Science and Technology Bureau of Zhejiang (Grant No. 2010C33124), and Municipal Science and Technology Bureau of Wenzhou (Grant No. H20080023).

Conflict of interest: None

\section{INTRODUCTION}

Maxillary central incisors are generally expected to erupt into the mouth around the age of 7 , following eruption of the mandibular incisors. An impaction must be suspected if the tooth fails to erupt into normal function within a specified time and the contralateral tooth has been present in the mouth for over a period of 6 months. ${ }^{1,2}$ Impaction of maxillary permanent incisors occurs in

\footnotetext{
${ }^{1,2}$ Department of Orthodontics, School of Stomatology Wenzhou Medical University, Wenzhou, Zhejiang Province City, China
}

Corresponding Author: Hu Rongdang, Professor, Department of Orthodontics, School of Stomatology, Wenzhou Medical University, No 113, West Xueyuan Road, Wenzhou, Zhejiang Province 325027, China, Phone: 8615057706297, e-mail: 353978071@qq.com
0,2 to $1 \%$ of the population. ${ }^{3,4}$ Although impaction of central maxillary incisors occurs less frequent than that of third molars and canines, patients often present to the dental clinic for management due to the high esthetic and functional concerns. ${ }^{1,5}$ The maxillary incisors are the most prominent teeth in an individual's smile, they are also the teeth that are on maximum display during speech in most individuals and the normal eruption, position and morphology of these teeth are crucial to facial esthetics and phonetics. ${ }^{5,6}$ The cause of impaction may be related to; presence of supernumerary teeth, cysts, trauma to the deciduous teeth and succedaneous tooth germ during development, unbalanced tooth-arch width relationships leading to loss of space in the arch, odontome and mucosal barriers in the path of eruption., ${ }^{1,-10}$

A detailed history, clinical and radiographic examination must be carried out by the clinician. Clinical examination may include assessment of the space available in the arch and bulge in the buccal or palatal side. An accurate diagnosis may be obtained with clinical and radiographic examination, such as panoramic radiograph, computed tomography (CT) and cone beam computed tomography (CBCT) ${ }^{3}$ This includes assessment of the morphology of the impacted tooth, position in the bone including height and both the vertical and horizontal angle of impaction.

The management of impacted maxillary incisors usually involves surgical exposure and the tooth left to erupt into alignment where there is enough space available in the arch with a favorable vertical and horizontal position or a combination of surgery and orthodontic traction. A simple appliance- the modified nance arch (MNA) appliance has been developed for orthodontic traction of difficult impacted teeth down into the arch. It is the most commonly used appliance in the Orthodontics Department-Wenzhou Medical University for the management of impacted incisors due to its relative ease of use and 

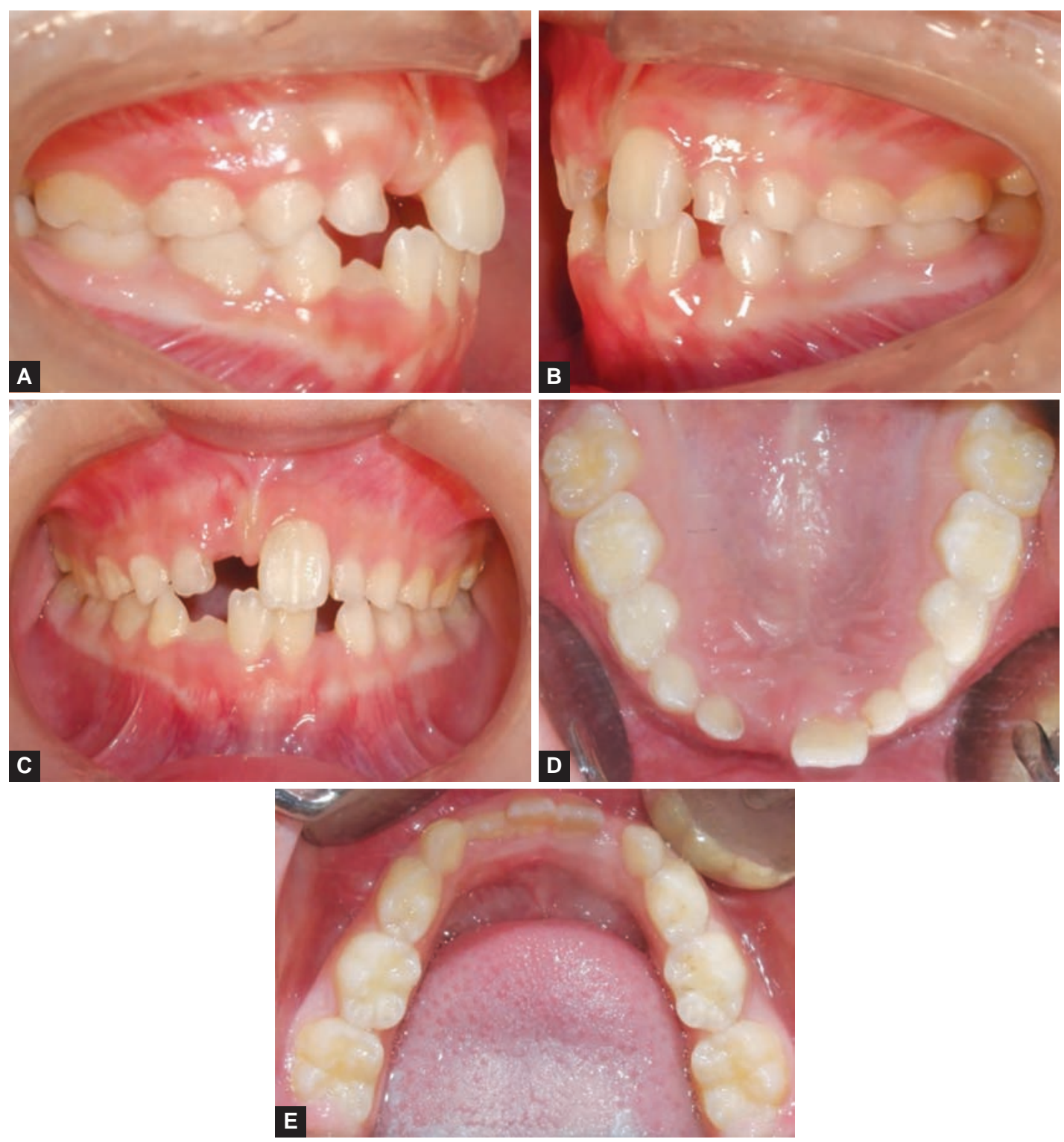

Figs $1 \mathrm{~A}$ to $\mathrm{E}$ : Intraoral views

good treatment results obtained. The appliance design consists of molar bands, transpalatal archwire and an extension arm which is adjusted by the clinician to the area of the tooth they wish to bring to alignment.

\section{CASE PRESENTATION}

An 8 years old girl presented to the dental clinic complaining of missing upper front tooth. Medical history was clear. Extraoral examination revealed average lower face height and no facial asymmetry. Temporomandibular joint was also normal with no clicks and no deviations on opening, closing or lateral excursions.

Intraoral examination (Figs 1A to E) revealed mixed dentition, missing upper right central incisor. Overjet and overbite were normal. The patient had moderate oral hygiene with good periodontal health and a slightly bulged alveolar ridge. The molar relationship was class 1 on both sides. A centreline shift was noted with the upper midline shifted $2 \mathrm{~mm}$ to the right and the lower midline coincident with facial midline.

Panoramic OPG and lateral cephalometric radiographs were taken (Figs 2A to C). No pathology noted, mixed dentition on a class 1 Skeletal pattern. Inverted position of the impacted incisor clearly seen on the lateral ceph radiograph. A CT scan was also taken for further assessment of the impacted tooth, which revealed an inverse impaction with the root apex on the palatal side and the crown labially positioned.

\section{TREATMENT PROGRESS}

The treatment plan included surgical exposure of the impacted incisor, orthodontic traction using MNA appliance, upper fixed appliance and retention. The window technique, which involved removal of a circular section of the overlying mucosa and the thin bony covering, was used to expose the incisor. Following surgical exposure 

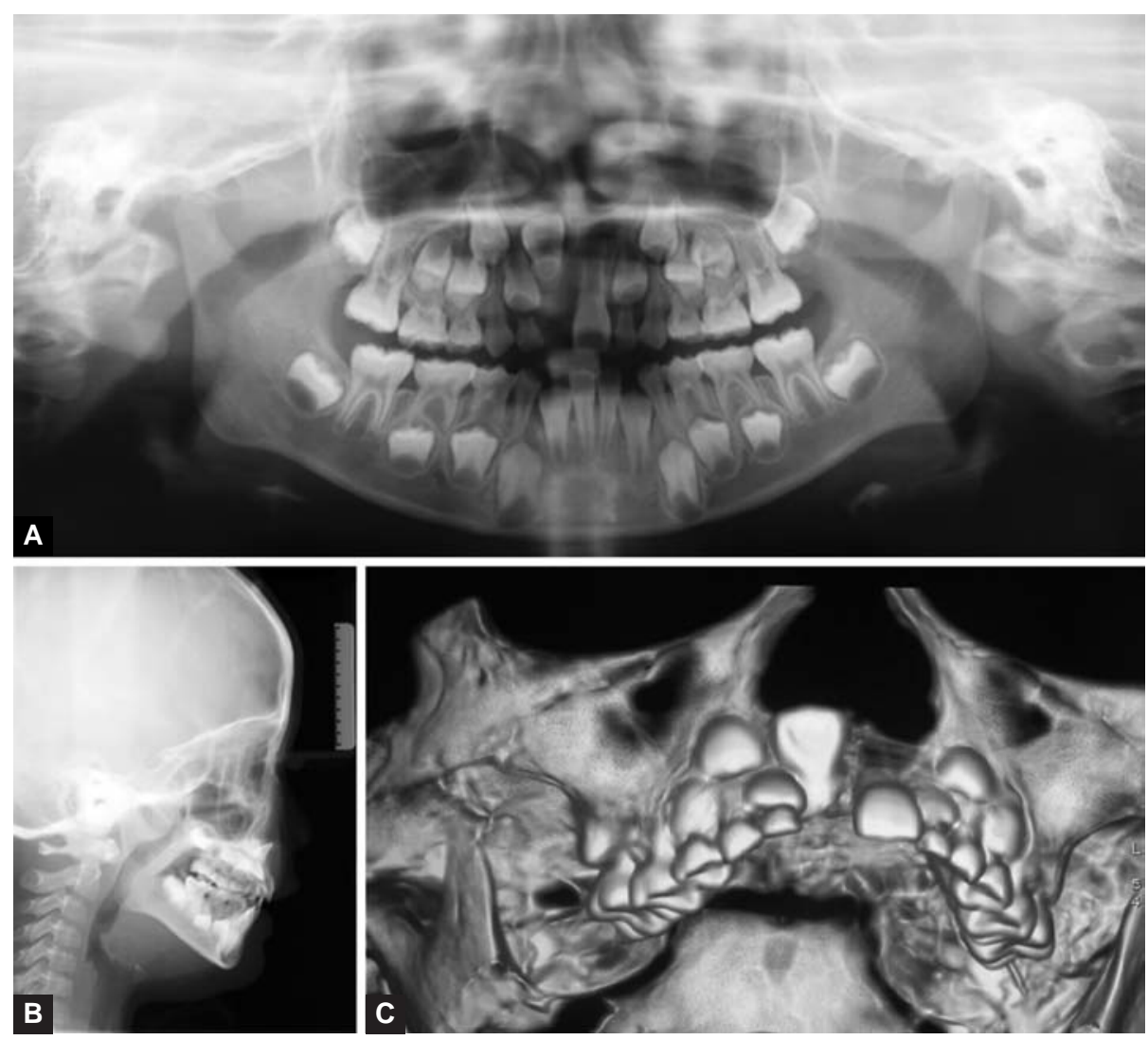

Figs 2A to C: Radiographic examination

of the tooth, an eyelet was placed on lingual surface of the crown of the impacted tooth. Modified nance arch appliance was cemented to the upper first molars and a 0.010 " wire ligature connected to the hook of MNA appliance used to initiate traction of the tooth. A modification was made by adding an acrylic ball to the extension arm hook to prevent irritation of the upper lip (Figs 3A to E). Initial review was carried out after 1 week for suture removal. An elastic line used to pull the tooth downwards in line of arch.

A lateral ceph was again taken showing the changed position (horizontal) of the impacted incisor (Figs 4A to D). Position and direction of pull changed by shortening the length of the extension arm and the acrylic ball removed so as not to interfere with the occlusion.

At 8 months review, the incisor pierced through the mucosa into the mouth (Figs 5A to F). Direction of force was changed to pull the tooth downwards and more palatally. Orthopantomogram (OPG) was also taken and showed position of the tooth relative to the left central incisor. Lateral ceph was also repeated.

Following a more downward movement of the incisor, preadjusted edgewise brackets were placed on all four upper incisors connected to the molar bands for alignment of the teeth; a phase two treatment.

Nickel-titanium (NiTi) coil spring was used to create space for the incisor. Elastic tie was used to apply a light force to pull the incisor down. Treatment was continued using flexible NiTi wire and later engaged the wire in the bracket slot for alignment into the arch (in the sequence: $0.014,0.016,0.018$ and 0.019 ") followed by 0.018 " stainless steel wire and finally $0.014 \mathrm{NiTi}$. The brackets removed after 1 year (from time of placement of the brackets) and a bonded retainer placed which was later removed.

Post-treatment pictures and radiographic analysis was again carried out at the end of treatment with the incisor in line with other teeth in the arch. Patient in permanent dentition and on the OPG mesial inclination of the lower second molars (more on the right side) was seen for which treatment was not provided to upright the teeth, oral hygiene especially for the posterior segment and need for monitoring reinforced (Figs 6A to F).

\section{DISCUSSION}

Several techniques have been used in the management of impacted incisors with the main objective of improving dental and facial esthetics, phonetics, establish good occlusion and enhance the health of the periodontium. ${ }^{1,4}$ Treatment varies from simple removal of the overlying primary tooth to allow for spontaneous eruption (where obstruction is due to over retained deciduous tooth), extraction of the impacted tooth followed by prosthetic replacement, surgical exposure alone or in combination with orthodontic traction and alignment depending on 

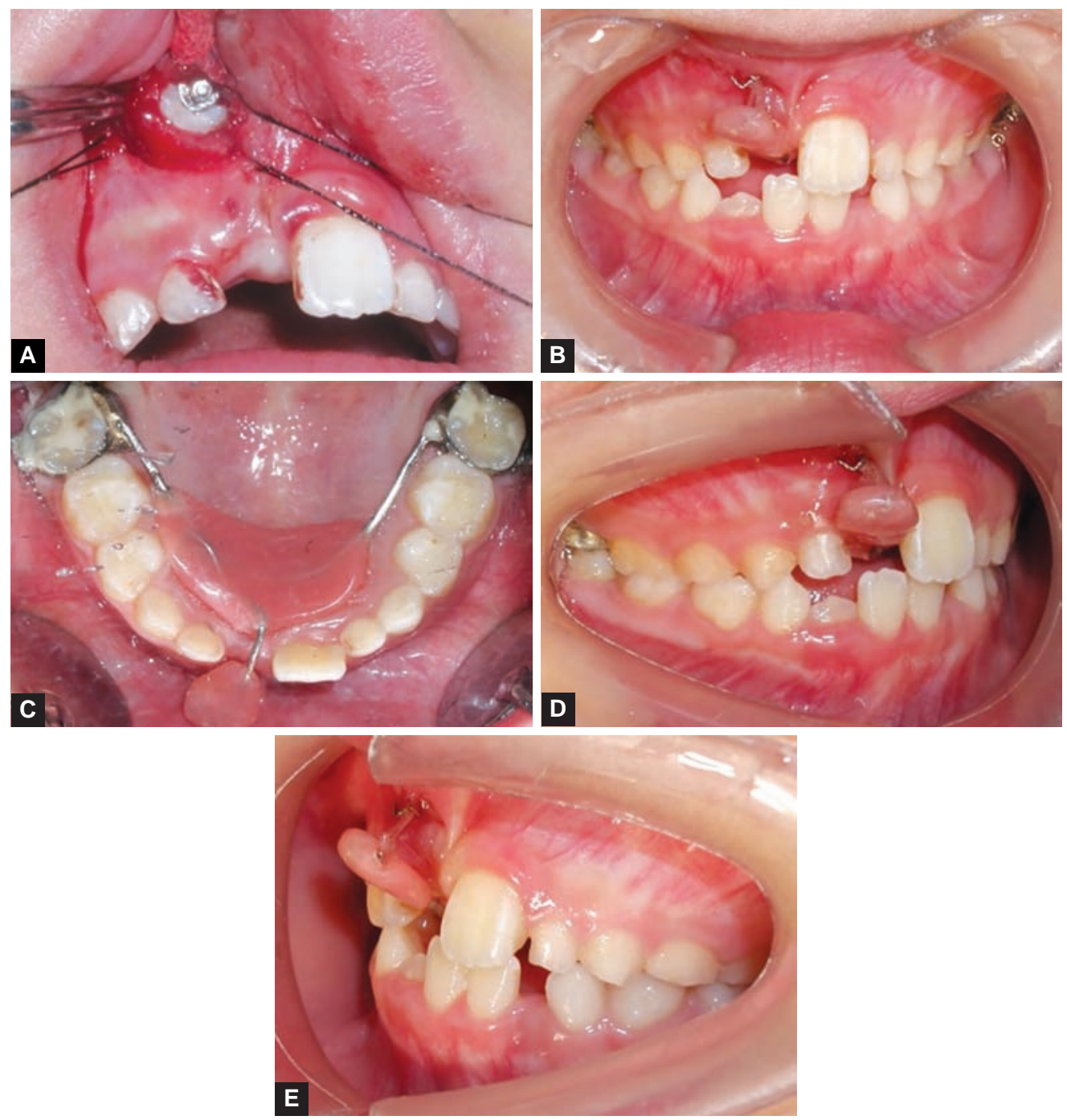

Figs $3 \mathrm{~A}$ to $\mathrm{E}$ : Phase 1 treatment-surgical exposure + MNA in place
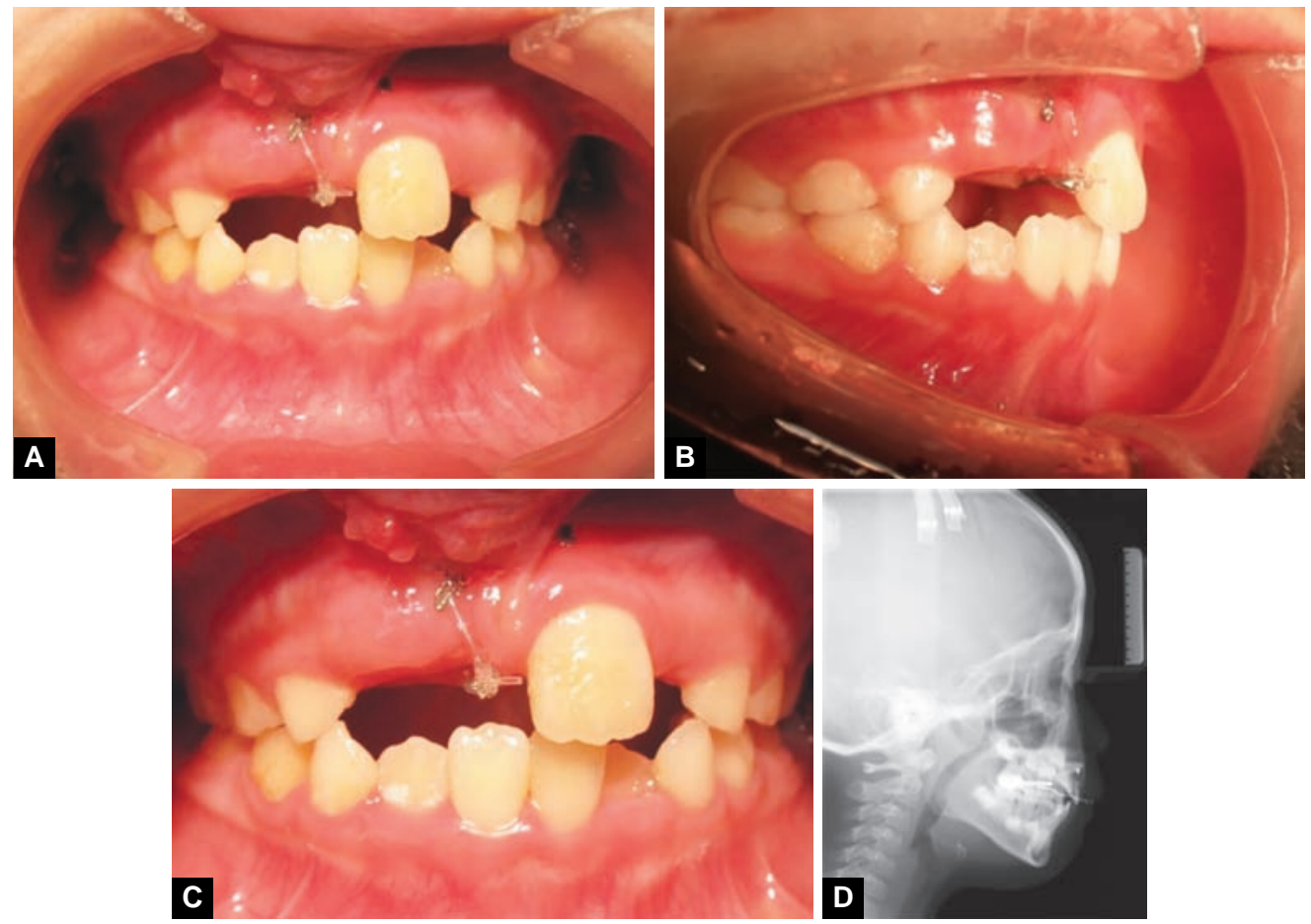

Figs 4A to D: Treatment progress-3 months review 

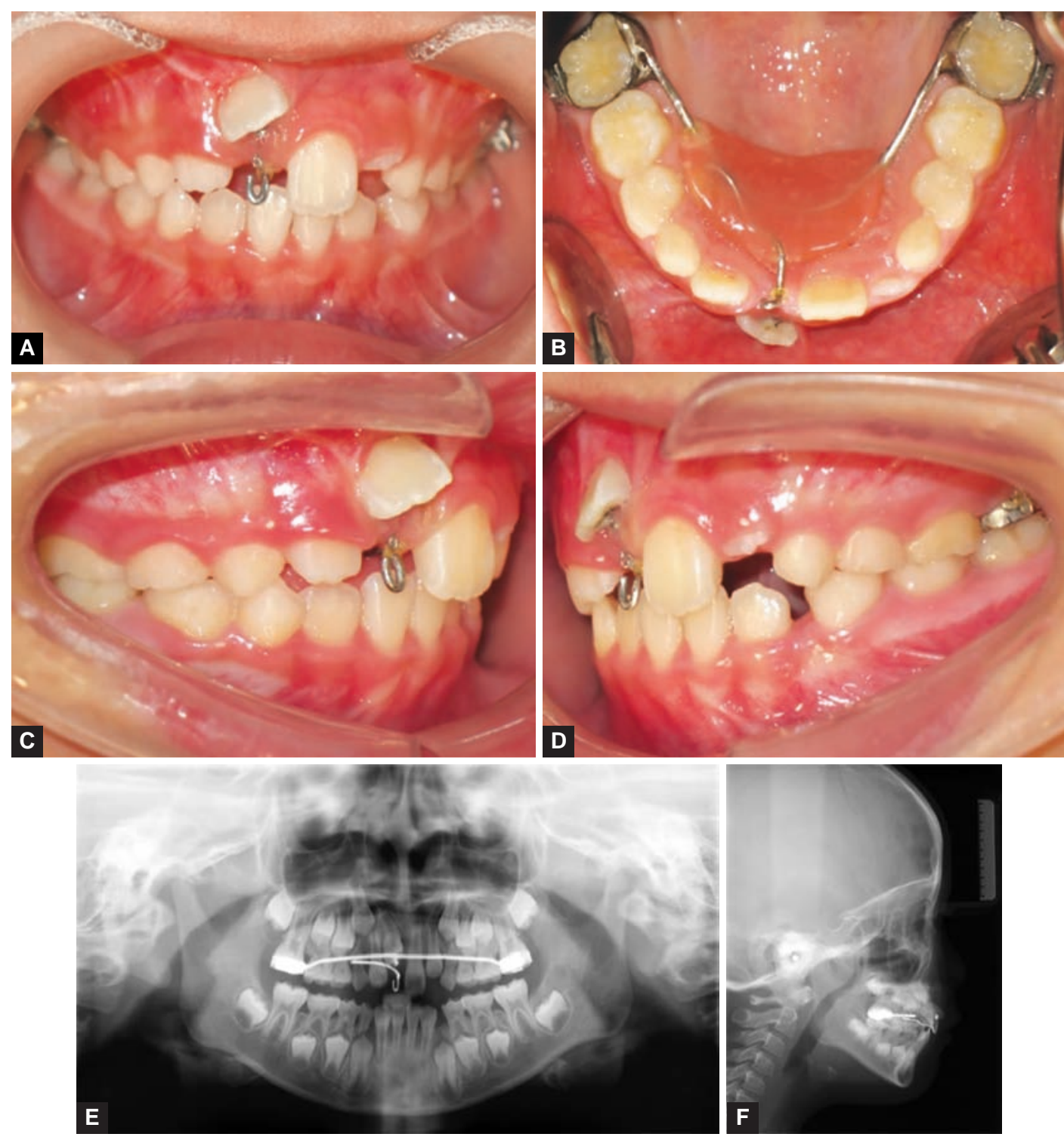

Figs 5A to F: Treatment progress-8 months review

the level and angle of impaction. In this case, surgical exposure was used in combination with orthodontic traction using MNA appliance since the vertical inverse position of the tooth, height and angulation were not favorable for unassisted eruption.

The use of MNA appliance requires a good clinical and radiographic examination prior to carrying out the treatment. A clinical examination must be carried out to determine the presence of a palatal or buccal bulge and availability of space for the unerupted incisor. Diagnosis of impacted tooth is verified and its location determined through radiographic evaluation, the most common being panoramic radiograph, $\mathrm{CT}$ and $\mathrm{CBCT}$. ${ }^{2,3}$ As reported by Becker $\mathrm{A}^{7}$ a lateral skull radiograph gives a more comprehensive picture of the tooth, particularly regarding details of its morphology, its height, and the overall orientation of its long axes. This was clearly seen from the pre-treatment lateral cephalometric radiograph. However, accurate localization in all three planes
(3D view) can be achieved through use of CT important in planning the type and position of the surgical exposure. Recently, CBCT has been introduced as a technique dedicated to the imaging of dental and maxillofacial structures and offers a higher advantage due to high sensitivity and lower radiation dose compared to conventional $\mathrm{CT}^{2,7}$

A MNA appliance, consisting of bands cemented on molars with a connecting palatal arch wire made of $1.2 \mathrm{~mm}$ stainless steel wire, palatal button made of acrylic resin and an extension arm made of $0.9 \mathrm{~mm}$ stainless steel wire was used for traction of the tooth. The extension arm; which has a small hook at the terminal end for attachment of ligature ties, passes through the space into the vestibular area. Its length can be adjusted in three-dimensional (3D) in the direction (occlusal, labiopalatal and mesiodistal) of desired movement and its length shortened as the tooth comes closer to alignment. As was seen in the case, 


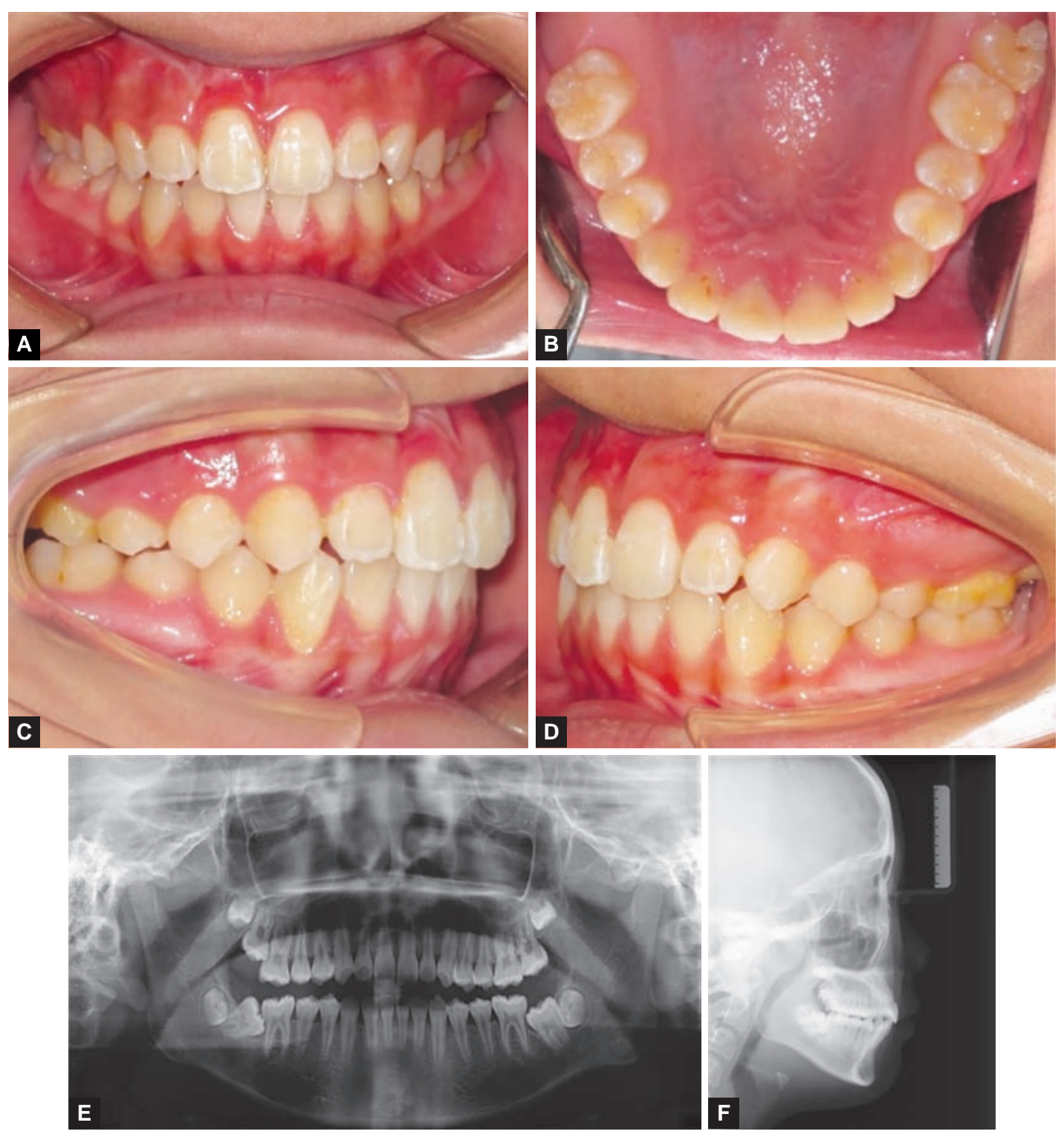

Figs 6A to F: Post-treatment records at 30 months

the hook was initially placed in the labial vestibule (Figs 3A to E) and position and length changed accordingly to move the tooth in the occlusal and palatal direction (Figs 4 and 5).

The use of MNA appliance has offered a greater advantage in increasing anchorage due to the use of a nance button and transpalatal archwire, thus eliminating reactionary intrusive forces on adjacent teeth. This is important in children with mixed dentition as there are not enough anterior permanent teeth for anchorage especially where the patient still has deciduous lateral incisors and canines. The traction force is another factor to be considered while aligning the impacted central incisor. Traction force ( 35 to $60 \mathrm{gm}$ ) can be adjusted vertical to the long axis of the tooth which reduces stress at the apex of the impacted incisor and cortical alveolar bone, hence reducing root resorption. This may also influence the length of clinical crown and post-alignment vitality. ${ }^{10}$
The use of light forces also has a high benefit with end treatment gingival appearance or contour. The limitation to the use of MNA appliance is that proper alignment and detailing of the arch and occlusion cannot be achieved through its single use where the teeth are not well aligned, thus necessitating the need for second phase of treatment using fixed appliances.

\section{CONCLUSION}

A combination of surgery and orthodontic traction using MNA appliance offers high advantages with respect to anchorage control and good periodontal and occlusal end results. Modified nance arch appliance is an easy appliance to use and highly tolerable by young patients. The movement of the impacted incisor through use of controlled force leads to improved esthetics and function with limited damage to adjacent teeth. 


\section{REFERENCES}

1. Kannan PK, Palanisamy SK, Kumar TS. A case of impacted maxillary central incisor and its management. J Pharm Bioallied Sci 2012;4(Suppl 2):S174-176.

2. Paoloni V, et al. Post-traumatic impaction of maxillary incisors: diagnosis and treatment. Ann Stomatol (Roma) 2013;4(2):174-183.

3. Pavoni C, et al. Impacted maxillary incisors: diagnosis and predictive measurements. Ann Stomatol (Roma) 2012;3(3-4): 100-105.

4. Pinho T, Neves M, Alves C. Impacted maxillary central incisor: surgical exposure and orthodontic treatment. Am J Orthod Dentofacial Orthop 2011;140(2):256-265.

5. Deshpande A, Prasad S, Deshpande N. Management of impacted dilacerated maxillary central incisor: a clinical case report. Contemp Clin Dent 2012;3(Suppl 1):S37-40.
6. Huber KL, Suri L, Taneja P. Eruption disturbances of the maxillary incisors: a literature review. J Clin Pediatr Dent 2008;32(3):221-230.

7. Becker A. The orthodontic treatment of impacted teeth. Informa Healthcare; UK: 2007.

8. Jones JW. A medico-legal review of some current UK guidelines in orthodontics: a personal view. Br J Orthod 1999;26(4):307-324.

9. Yeluri R, et al. Multiple supernumerary teeth associated with an impacted maxillary central incisor: surgical and orthodontic management. Contemp Clin Dent 2012;3(2): 219-222.

10. Kalaskar RR, Kalaskar AR. Multidisciplinary management of impacted central incisors due to supernumerary teeth and an associated dentigerous cyst. Contemp Clin Dent 2011;2 (1):53-58. 